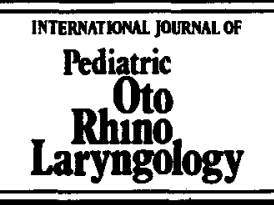

\title{
The click-evoked oto-acoustic emission, c-EOAE, in preterm-born infants in the post conceptional age range between 30 and 68 weeks
}

\author{
Bert G.A. van Zanten*a, M. Renée Kok ${ }^{\mathrm{a}}$, Michael P. Brocaar ${ }^{\mathrm{a}}$, \\ Pieter J.J. Sauer ${ }^{b}$ \\ "Dept of ENT/Audiology, Erasmus University Hospital/Sophia Children's Hospital, Rotterdam, \\ The Netherlands \\ ${ }^{b}$ Dept of Neatology, Erasmus University Hospital/Sophia Children's Hospital, Rotterdam, \\ The Netherlands
}

\begin{abstract}
Click-evoked otoacoustic emissions (cEOAEs) were repeatedly recorded in an operational sample of 144 very low birth weight (VLBW) infants. A subgroup of 22 was composed of all those babies in which at least 4 recordings were successfully done. The mean birth weight of this group was $1040 \mathrm{~g}$, and the mean duration of assisted ventilation was 17 days. The OAE-recordings were done in the post conceptional age (PCA) range between 30 and 68 weeks. In relation to ear function screening it was shown that the EOAE was present in $95 \%$ of the ears at least once at any age, while it was present in all recordings in only $34 \%$. From a longitudinal analysis of the recordings per infant it appeared that: (1) the OAE recorded was already present in one infant at the PCA of 29.4 weeks; (2) in most infants the level of the OAE varies strongly between recordings; (3) in each infant the OAE-level shows an increase with age, on average this growth amounts to $10 \mathrm{~dB}$ between the PCAs of 31 and 42 weeks; (4) there is no clearcut difference in the growth of high-and low-frequency components of the EOAE.
\end{abstract}

Keywords: Oto-acoustic emission; Very low birthweight babies; Preterm-born infants

* Corresponding author.

0165-5876/95/\$09.50 $\bigcirc 1995$ Elsevier Science Ireland Ltd. All rights reserved SSDI 0165-5876(94)01158-8 


\section{Introduction}

It is clear from this issue (see contributions by Eggermont and Pujol) that the ear is already functioning in the preterm period, although it has not fully matured. We wondered whether the phenomenon of click-evoked otoacoustic emission would somehow reflect the maturation of the ear in the preterm period. Previously papers have been published only on the application of c-EOAEs for hearing screening of the neonatal intensive care unit population $[10,13]$. Below we report on repeatedly recorded c-EOAEs and their development in preterm-born infants.

\section{Subjects}

In this study babies were included that were born with a very low birth weight (VLBW), i.e. a weight not exceeding $1500 \mathrm{~g}$, and after at maximum 37 weeks of gestation. Before their c-EOAEs could be recorded they also had to be stable enough, in the neonatologist's judgment, and without assisted ventilation. Babies were totally excluded if at higher risk for hearing impairment due to a positive family history, cranio-facial malformations, or a syndrome associated with hearing loss.

For the purpose of this study we aimed at weekly recording of c-EOAEs in each baby during their stay in the ward and at 6,13 , and 26 weeks of corrected age in the outpatient clinic. However recording was in no case attempted before the age of 4 days, because at lower ages the level of the emission and its prevalence in the newborn still grows $[5,6]$.

We examined in total 144 individuals, but only in 22 individuals did we succeed in recording four or more times. Six of these 22 were female. The range of birth weights was between 720 and $1410 \mathrm{~g}$, with a mean value of $1040 \mathrm{~g}$. The range of gestational ages was between 25.3 and 32.0 weeks (mean 28.1 weeks). A scatterplot of birth weight against gestational age is shown in Fig. 1. All but two babies were intubated for assisted ventilation during at least 1 day and up to 56 days, on average 18 days. Six infants went through a sepsis, but none had meningitis.

\section{Methods}

\subsection{Clinical patient data}

For each individual the following perinatal data were recorded: birth weight, gestational age, Apgar scores, and umbilical $\mathrm{pH}$. As data relevant to patient history prior to testing, the administration of ototoxic drugs and the maximum serum level of bilirubine were recorded. At testing the age and the weight were recorded, as well as the administration of extra oxygen and the presence of a naso-oesophageal tube for feeding. No attempt at systematic otoscopy or BERA was made.

\subsection{Equipment}

c-EOAE-recording was done with the only available set of equipment at the time of the start of this study. This was the ILO88 operating with software version 3.0 


\section{Birthweight against Gestation}

in 22 VLBW-infants

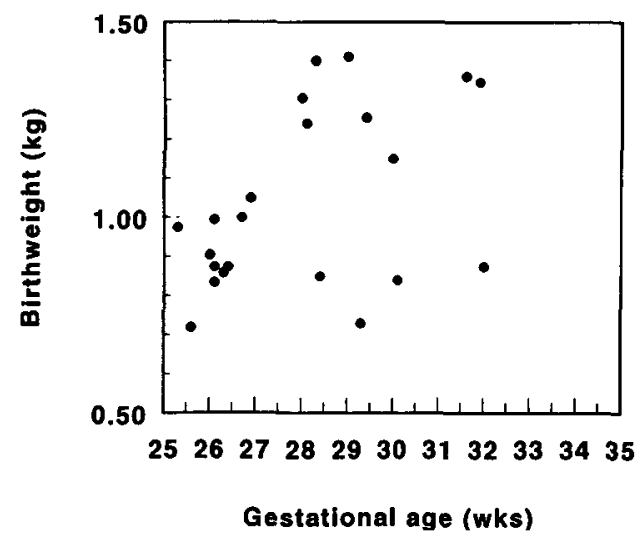

Fig. 1. Scatterplot of birth weight against gestational age of the individuals examined. The average weight was $1040 \mathrm{~g}$ and the average duration of gestation 28 weeks.

(OtoDynamics, UK). The probes used were the old and later the new model of the baby-probe that came with the apparatus. The equipment was used in its standard setting, which means that the nonlinear component of the oto-acoustic response to click stimulation is averaged out of 260 non-linear stimulus sequences of 4 clicks. It is generally accepted that this nonlinear component represents cochlear activity only and is free of middle ear artefact. All recordings were stored on disk for later processing. During recording the oto-acoustic signal is passed through an artefactrejector and highpass filtered with a cutoff frequency of $500 \mathrm{~Hz}$. In order to reduce low-frequency noise contamination of the responses even more, we highpass filtered all responses off-line with a cutoff frequency of $1 \mathrm{kHz}$.

\subsection{Response-scoring}

We subjectively scored each of the recorded otoacoustic response waveforms as to the presence of cochlear activity in three categories: 'present', 'doubtful', or 'absent'. This judgement was based on visually estimated reproducibility of the waveform, or part of it, and the presence of spectral response energy in excess of that of the background noise.

\subsection{Recording}

No time-constraints were set to the examiner (2nd author), as screening was not the primary goal of this study. The first recordings in all but three infants were done while they were in an incubator. During recording the incubator's ventilator had to be switched off and the doors closed in order to get a low environmental noise level, which is necessary to receive enough otoacoustic responses for the artefact rejecting averager. Later recordings were done while the infants were in a crib, or lying on one of their parents' lap or in a baby chair. 
As previously reported [11-13], it proved much more difficult to do c-EOAErecording in the preterm group than in healthy newborns, especially for the follow-up exams at the outpatient clinic. Therefore, it was recorded whether or not a c-EOAE-recording attempt was technically succesful. A recording attempt was considered succesful if 260 sweeps had been accepted, or if a clear c-EOAE was judged to be present in the result. If the c-EOAE was judged to be absent, the recording attempt was scored succesful only if the stimulus level had been over 72 dBSPL.

\subsection{Data-processing}

Age. Up to 43 weeks post-conceptional age (PCA) at testing was rounded to weeks; if higher it was assigned to PCA-groups of 44-48, 49-59, and 59-65 weeks.

Success-rate of otoacoustic response recording. This enabled the calculation of the 'success rate' of recording in a specific age-class, defined as the percentage of recording attempts that were technically successful.

$c-E O A E$-presence. For each specific age class the percentage of recordings that showed cochlear activity were calculated, by dividing 100 times the number of 'c-EOAE-present' recordings by the total number of successful recordings in that age class. c-EOAE-presence as a percentage might also be viewed as a kind of c-EOAE-prevalence per age class. However this is not really the case, because of the small sample and the fact that one individual ear was included in various age classes.

c-EOAE-level. The ILO88 gives as one of its results the sound pressure level of the otoacoustic response waveform. Another result is the spectrum of the response. From this spectrum we calculated the emission level for two subbands of frequencies, from 1000 up to $2350 \mathrm{~Hz}$ and above that limit (up to the recording limit of $6250 \mathrm{~Hz}$ ). For those responses that were scored 'c-EOAEpresent', so judged to show cochlear activity, the c-EOAE-levels were taken and averaged per age class. As appropriate we fitted these data with simple growth functions of the form level (age) $=\mathrm{L}_{0}\left\{1-\exp \left[\left(a_{0}-\right.\right.\right.$ age $\left.\left.) / \theta\right]\right\}$, where $\mathrm{L}_{0}$ is the mean level at which the emission for this group of 22 infants saturates (the matured level), $a_{0}$ is the postconceptional age at which the emission level has grown to $0 \mathrm{dBSPL}$, and $\theta$ is the time-constant of growth. Three times this constant equals the growth period between $0 \mathrm{dBSPL}$ and $95 \%$ of the matured level. Adding this growth period to $a_{0}$ gives the PCA at which the emission level reaches $95 \%$ of the saturation level.

In order to create a good overview of all responses recorded in each individual infant, we wrote a turbo-Pascal program that processed the set of datafiles for an ear into a pattern of responses, in which they are sorted by age at recording time.

\section{Results}

As examples of the filtered serial recording results, Fig. 2 shows two series of recordings made at different post-conceptional ages in the left ears of two infants. 
For all 22 infants Fig. 3 shows both the success rate of recording and the 'c-EOAE-presence' in various age classes. For the recordings that were scored as 'c-EOAE-present', the mean level of the c-EOAE was calculated as a function of

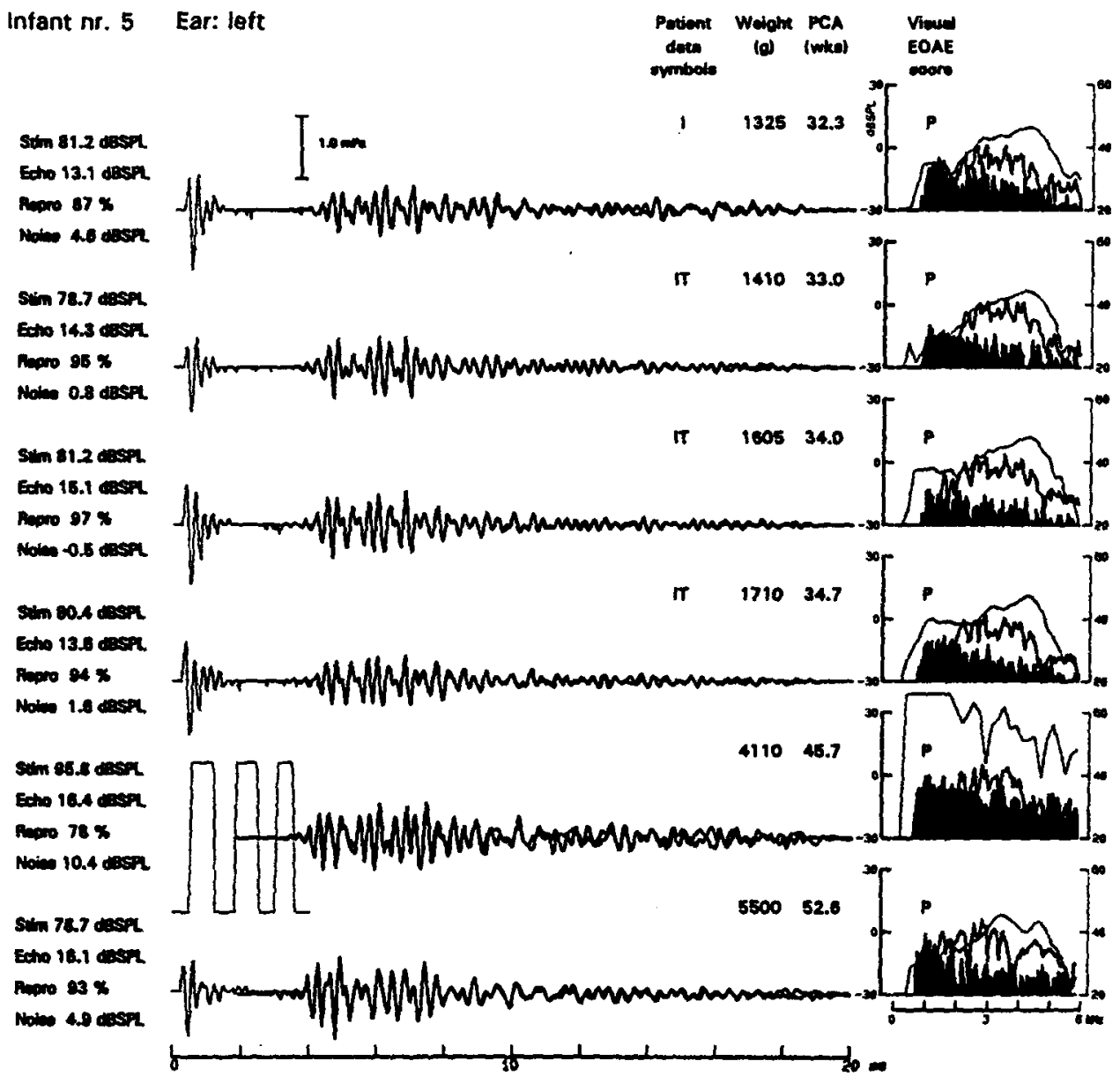

Fig. 2. Two examples of serial recording patterns with responses downwardly sorted by PCA at the time of recording. The left pattern shows the results for the right ear of infant number 5 , the right panel shows results for the left ear of infant number 11. The large middle part of each pattern show with solid lines the response waveforms (labeled ' $A$ ' and ' $B$ ' in the ILO88), with vertically the sound pressure amplitude and horizontally the post-stimulus time. The dotted line starting at $B$ ms shows the stimulus waveform used during the recording of the response. To the right of each response the spectra of the response, the background noise, and the stimulus are given by a thick solid line, a black shaded area, and a dotted line, respectively. To the left of each waveform annotation gives the stimulus sound pressure level, that of the response, the reproducibility of the response, and the level of the background noise in the response. PCA and weight at the time of recording are given at each waveform at about 19 and 17 $m$ s post stimulus time. The subjective visually made 'EOAE-present' or 'EOAE-absent' scores are symbolized with 'P' or 'A' respectively, in the spectra at about $1 \mathrm{kHz}$. 


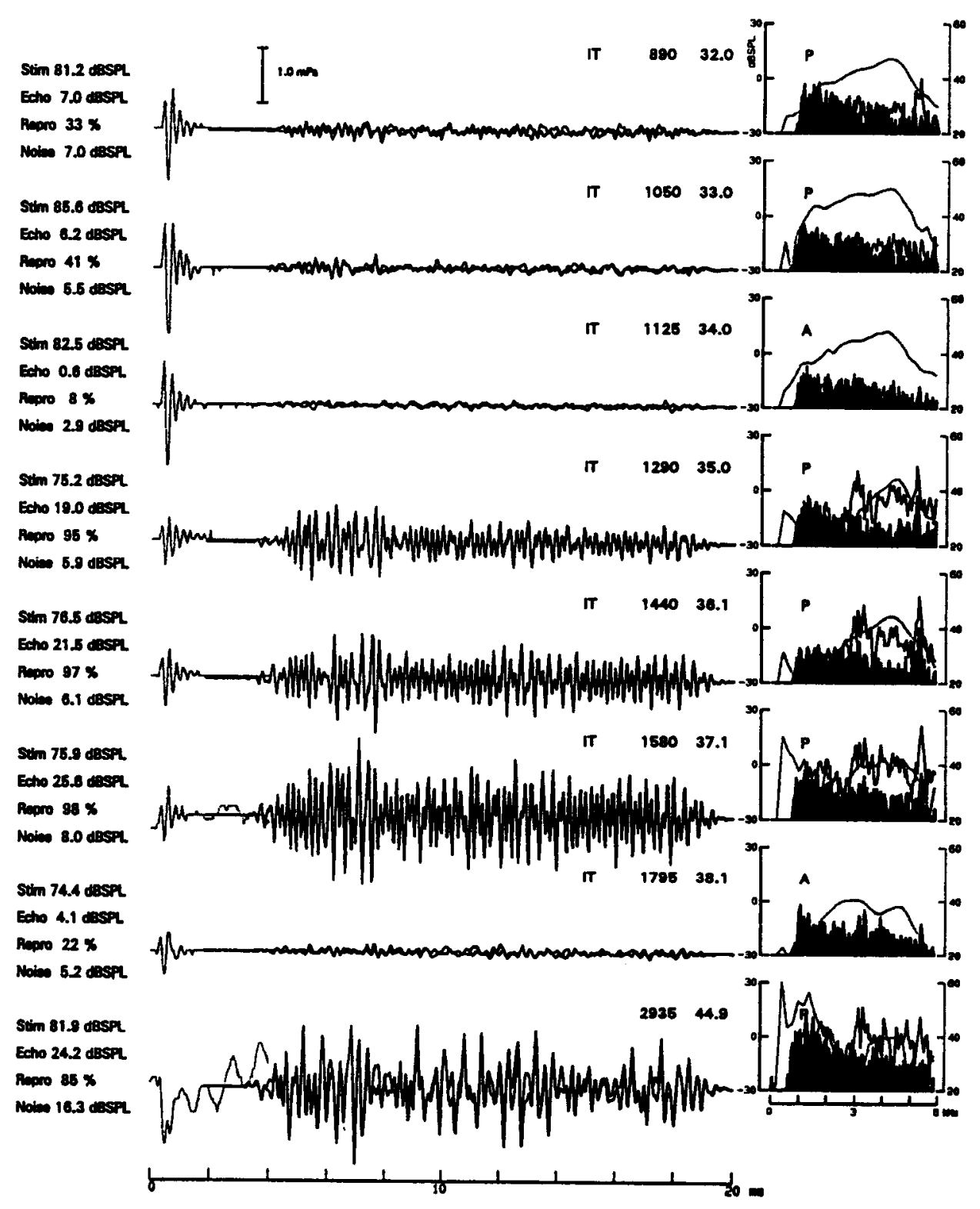

Fig. 2 (continued). 


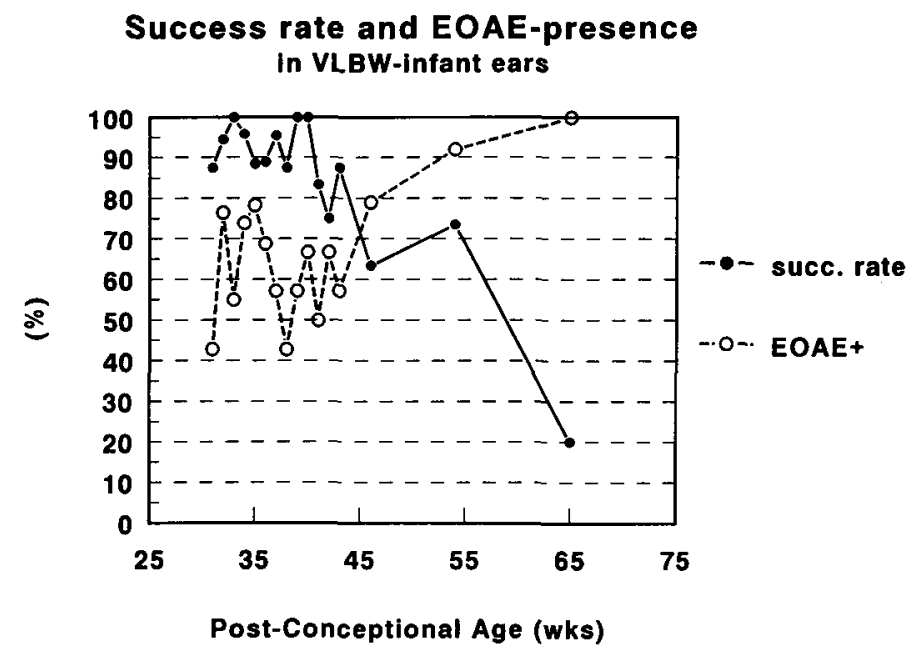

Fig. 3. The success rate of recording, defined as the percentage of recording attempts in a specific age-class that were considered as technically successful, plotted against age at recording time with dots connected by a solid line. The dashed line connecting the circles shows the percentage of successful recordings with an 'EOAE-present' score.

age and is given as a solid line in Fig. 4. The level is also given for two subbands of the emission energy, i.e. between 1000 and $2350 \mathrm{~Hz}$ and between 2350 and $6250 \mathrm{~Hz}$. In the table in Fig. 4 the results of the fits with the simple mathematical growth functions are given. For the three frequency bands the correlation between the fitted functions and the data is given in the second column, the standard error of estimate in the third column, the saturation levels in the fourth column, and the age at which the emission level has about matured, age- $95 \%$, in the last column. We also extrapolated the function down to the age (fifth column) at which the emission in each frequency band would have reached $3 \mathrm{dBSPL}$, viewing this as a detection limit level, below which the emission goes undetected with the recording techniques used.

\section{Discussion}

The neonatal high and intensive care wards of the Sophia Childrens's Hospital form a tertiary referral centre for a population of about 3 million. Infants admitted either have an extremely low birth weight (below $1000 \mathrm{~g}$ ) or are critically ill. Generally they are transferred to neonatal wards in other hospitals as soon as their condition allows. This fact is the main cause for the low yield of long-term followed-up individuals. Moreover, attendance at our follow-up outpatient clinic was only moderate, due to the fact that most infants were also under control of a pediatrician in a local hospital. 


\section{Growth of EOAE-Level (dBSPL) low/high frequency bands}

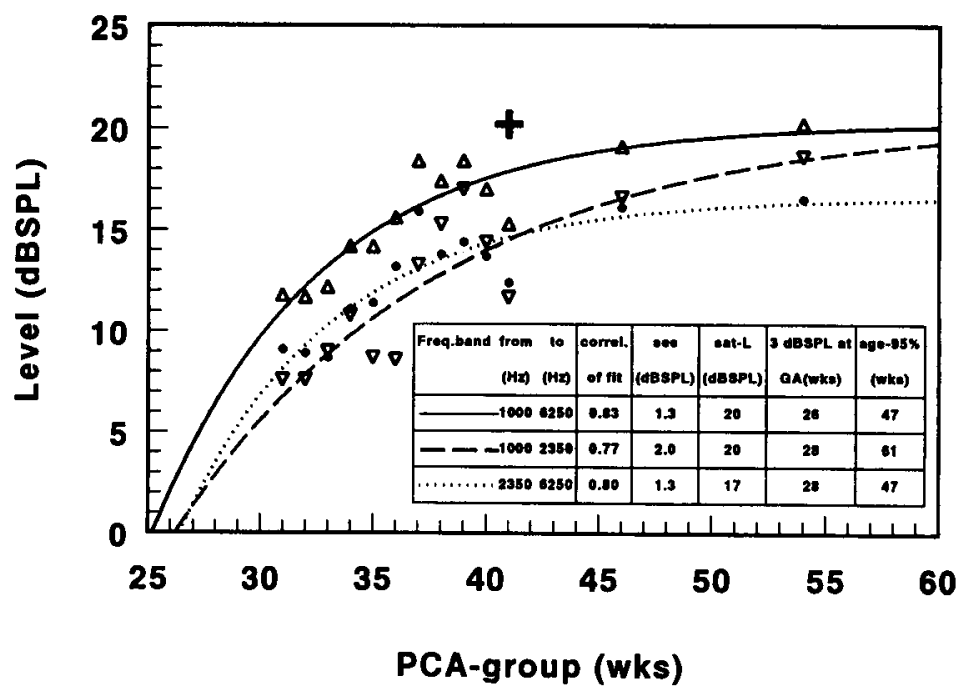

Fig. 4. The mean level of the responses with an 'EOAE-present' score given for various age classes. The upwardly pointing triangles denote the level of the response filtered between 10 and $62 \mathrm{~Hz}$, the downwardly pointing triangles indicate emission level for frequencies between 10 and $2350 \mathrm{~Hz}$, the dots for frequencies between 23 and $62 \mathrm{~Hz}$. The curves are simple saturating growth functions fitted on the data. The results for the fittings are given in the inset table (see text).

Groups of neonatal intensive care unit (NICU) babies can be very different between studies, because of the fact that a baby's chances of being transferred to a NICU is highly dependant on local habits and NICU availability. In order to enable a better comparison we limited inclusion to VLBW babies and gave a limited set of descriptive data for these 22 infants, not only on birth weight and gestation but also on the duration of assisted ventilation and the incidence of sepsis.

The series of recordings shown in Fig. 2 for infant number 5 is typical for about one-third of the infants. The c-EOAE in infant 5 was present at our first recording, in this case at a PCA of 32.2 weeks. The level of the emission grows gradually up to a stable level at the age of 45.7 weeks. In one individual we found a clearly present and broadband emission at a PCA of 29.4 weeks. So the emission already can be detected early in premature life.

The other example in Fig. 2, the series recorded with infant 11, is typical for about half of the cases. The first recording attempt at a PCA of 31.4 weeks technically failed in the right ear. The emission was scored as present, albeit weak, at weeks 32 and 33 , absent at 34, present again and growing in strength at weeks $35,36,37$, absent again at week 38 , and present again at the first (and unfortunately last) outpatient clinic follow-up recording at 46 weeks. The matured emission level, about $25 \mathrm{dBSPL}$, is well above group average, which can be attributed to the 
fact that the emission's waveform is dominated by a spontaneous emission [7]. This series of recordings is typical in the sense that the emission can suddenly disappear and reappear. In this VLBW population the emission presence in an individual ear is often unstable. We think this instability is due to the high prevalence of (transient) middle ear dysfunctions in the VLBW population [1-3].

This instability is also the reason why for the group as a whole the emission presence in any PCA-class below 40 weeks averages at only about $60 \%$ (see Fig. 3). This would have been the 'pass-rate' in a screening approach of this group, so with only one recording session per infant. Overall we found an emission in all but one individual $(95 \%)$ due to the repeated recording approach. (This individual's BERA showed no cochlear damage, while a small conductive sensitivity loss could not be excluded at a PCA of 46 weeks). In relation to c-EOAE-screening, Fig. 3 also shows that the emission presence strongly increases up to almost $100 \%$ with PCA above 40 weeks. While this increase in emission presence augments the feasibility of screening in this VLBW group, its effect is completely negated by the strong decrease in the technical success rate of recording in that age range. This same counteracting effects of emission prevalence and success rate of recording on the feasibility of ear-screening with the c-EOAE was previously reported for a much larger group in VLBW-infants [8].

The growth of the mean emission level during maturation is shown to follow a simple saturating growth function (see solid line in Fig. 4). From the fitted growth function we can conclude that the maturational growth takes on average up to about 47 weeks after conception. With the c-EOAE-recording techniques used in this study the emission is probably detectable already in infants with a PCA of 26 weeks. We were unable to control this prediction because of a lack of infants of that age.

We attempted to analyse recording series for the individual infants as to the systematic change in frequency composition with age. From that qualitative analysis the impression resulted that first the higher frequency energy comes up and a little later the lower frequency energy. However, our quantitative analysis was not conclusive. The growth functions fitted for the emission level due to frequencies above or below $2350 \mathrm{~Hz}$ (dotted and dashed line in Fig. 4, respectively), indeed reveal a tendency for the high frequency band of the emission to grow faster than the lower frequency band, which results in a higher age- $95 \%$ for the low-frequency band, 61 vs. 47 . But the difference in growth rate was statistically non-significant. The scatter of data around the fitted curves for the 'lower' and 'higher' frequency bands is too large. This non-significance applies also to the difference in saturation levels for the 'lower' and 'higher' emission frequencies.

The matured mean emission level in this group of VLBW-infants, $20.2 \mathrm{dBSPL}$, is equal to that found in the healthy newborn, as indicated by the large + symbol in Fig. 4 [6]. One must keep in mind however that the inter-individual variation of the matured emission strength is considerable. For example the emissions of infant 5, shown in Fig. 2, saturate at $4 \mathrm{~dB}$ below the mean and of infant 11 at $5 \mathrm{~dB}$ above. The large inter-individual variation of the emission strength was previously reported for healthy newborns and for normal hearing adults $[4,6,9]$. 
We are tempted to believe that the shown maturation of the emission is mirroring the maturation of the cochlea. However, on the basis of the present data we cannot exclude a maturational contribution by the middle ear. As discussed above, we view the middle ear, due to its frequent transient dysfunctions in the VLBW population, as a strong confounding factor in recording the maturation of the emission. To our knowledge, methods to measure the middle ear characteristics in infants at age below 46 weeks are lacking at present.

\section{Conclusions}

The click-evoked otoacoustic emission was already detected in a VLBW infant at the post-conceptional age of 29.4 weeks and will probably be detectable from the age of 26 weeks with the present emission recording methods. The maturation of the level of the click-evoked otoacoustic emission in VLBW infants is completed at a post-conceptional age of about 46 weeks. Although not statistically significant, the higher frequencies in the otoacoustic emission seem to mature faster than lower frequencies. The mean emission level in VLBW saturates at $20.2 \mathrm{dBSPL}$, the same strength as found in healthy newborns.

\section{Acknowledgments}

We gratefully acknowledge the generous support of this study by the Sophia Foundation for Scientific Research and by the Heinsius Houbolt Foundation.

\section{References}

[1] Balkany, T.J., Berman, S.S., Simmons, M.A. and Jafek, B.W. (1978) Middle ear effusions in neonates. Laryngoscope $88,398-405$

[2] Eggermont, J.J. and Salamy, A. (1988) Maturational course for the ABR in preterm and full term neonates. Hear. Res. 33, 35-48.

[3] Jacobson, J.T. and Morehouse, C.R. (1984) A comparison of auditory brain stem response and behavioural screening in high risk and normal newborn infants. Ear Hear. 5, 247-253.

[4] Kemp, D.T. (1978) Stimulated acoustic emissions from within the human auditory system. J. Acoust. Soc. Am. 64, 1386-1391.

[5] Kok, M.R., Van Zanten, G.A. and Brocaar, M.P. (1992) Growth of evoked oto-acoustic emissions the first days post partum: a preliminary report. Audiology 31, 140-149.

[6] Kok, M.R., Van Zanten, G.A. and Brocaar, M.P. (1993a) Click-evoked oto-acoustic emissions (EOAEs) in 1036 ears of healthy newborns. Audiology 32, 213-224.

[7] Kok, M.R., van Zanten, G.A. and Brocaar, M.P. (1993b) Aspects of spontaneous otoacoustic emissions in healthy newborns. Hear. Res. 69, 115-123.

[8] Kok, M.R., van Zanten, G.A., Brocaar, M.P. and Jongejan, H.T.M. (1994) Click-evoked otoacoustic emissions in very-low-birth-weigth infants: a cross-sectional analysis. Audiology 33, $152-164$.

[9] Probst, R., Lonsbury-Martin, B.L. and Martin, G.K. (1991) A review of otacoustic emissions. J. Acoust. Soc. Am. 89, 2027-2067.

[10] Stevens, J.C., Webb, H.D., Smith, M.F., Buffin, J.T. and Ruddy, H. (1987) A comparison of oto-acoutic emissions and brain stem electric response audiometry in the normal newborn and babies admitted to a special care baby unit. Clin. Physiol. Meas. 8, 95-104. 
[11] Stevens, J.C., Webb, H.D., Hutchinson. J.. Connell, J., Smith, M.F. and Buffin. J.T. (1989) Click evoked otoacoustic emissions compared with brainstem electric response. Arch. Dis. Child. 64. $1105-1111$.

[12] Uziel. A. and Piron. J.P. (1991) Evoked otoacoustic emissions from normal newborns and babies admitted to an intensive care baby unit.

[13] Webb, H.D. and Stevens, J.C. (1991) Auditory screening in high risk neonates: selection of a test protocol. Clin. Phys. Physiol. Meas. 12. 7586. 